\title{
Laser ultrasonic testing for near-surface defects inspection of 316L stainless steel fabricated by laser powder bed fusion
}

\author{
Ting Dai ${ }^{1}$, Xiao-jian Jia', Jun Zhang ${ }^{2}$, Jin-feng $\mathbf{W u}^{3}$, Yi-wei Sun ${ }^{1}$, Shu-xian Yuan ${ }^{2}$, Guan-bing Ma ${ }^{1,3}$, Xiao-jing Xiong ${ }^{4}$, \\ *Hui Ding ${ }^{1}$ \\ 1. School of Materials Science and Engineering, Southeast University, Nanjing 211189, China \\ 2. School of Power and Mechanical Engineering, Wuhan University, Wuhan 430072, China \\ 3. CGN Inspection Technology Co., Ltd., Suzhou 215100, Jiangsu, China \\ 4. Monash (Suzhou) Engineering Technology Co., Ltd., Suzhou 215123, Jiangsu, China
}

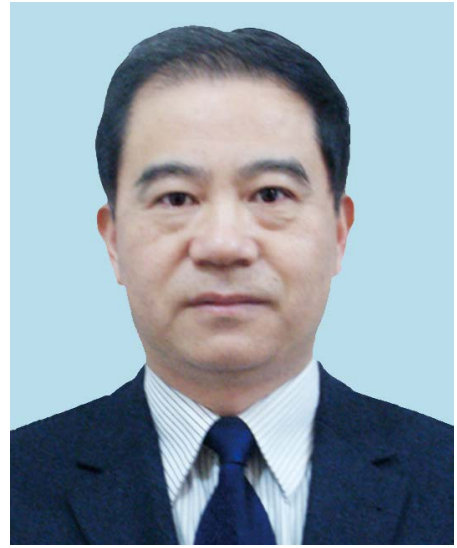

*Hui Ding

Chief Professor. His research interests mainly focus on the computational materials science, special structural material damage, and nondestructive testing technology. Prof. Ding is a Member of the Advanced Manufacturing Department of Science \& Technology Commission of Ministry of Education. He has presided over a number of national key research and development projects such as "High-frequency Ultrasonic Testing Technology and Equipment for Metal Additive Manufacturing". He won the second prize of the National Science and Technology Progress Award in 2017.

E-mail: hding@whu.edu.cn

\begin{abstract}
The laser powder bed fusion (L-PBF) method of additive manufacturing (AM) is increasingly used in various industrial manufacturing fields due to its high material utilization and design freedom of parts. However, the parts produced by L-PBF usually contain such defects as crack and porosity because of the technological characteristics of L-PBF, which affect the quality of the product. Laser ultrasonic testing (LUT) is a potential technology for on-line testing of the L-PBF process. It is a non-contact and non-destructive approach based on signals from abundant waveforms with a wide frequency-band. In this study, a method of LUT for on-line inspection of L-PBF process was proposed, and a system of LUT was established approaching the actual environment of on-line detection to evaluate the method applicability for defects detection of L-PBF parts. The detection results of near-surface defects in L-PBF $316 \mathrm{~L}$ stainless steel parts show that the cracktype defects with a sub-millimeter level within $0.5 \mathrm{~mm}$ depth can be identified, and accordingly, the positions and dimensions information can be acquired. The results were verified by X-ray computed tomography, which indicates that the present method exhibits great potential for on-line inspection of AM processes.
\end{abstract}

Key words: additive manufacturing; $316 \mathrm{~L}$ stainless steel; on-line inspection; laser ultrasonic testing; non-destructive testing

CLC numbers: TG142.71; Document code: A; Article ID: 1672-6421 2021 04-360-09

\section{Introduction}

Additive manufacturing (AM) technology features the accumulation of the materials layer by layer to realize the manufacturing of three-dimensional (3D) solid parts. This technology mainly consists of powder bed fusion (PBF) and directed energy deposition (DED) ${ }^{[1,2]}$. However, in a real production environment, yield rate and quality control are still among the primary challenges for AM. The unavoidable defects such as pores, cracks, and lack of fusion in AM parts are caused by the existence of insufficient melting and high thermal stress during the AM process ${ }^{[3-5]}$. The lack of efficient and non-destructive evaluation methods for AM products has become a major bottleneck restricting the development and application of AM technology.

The non-destructive testing methods of parts fabricated by AM include on-line testing and off-line testing. For off-line testing, X-ray computer tomography (X$\mathrm{CT}$ ) and ultrasonic testing (UT) are often used after parts are completed by AM ${ }^{[6,7]}$. Rometsch et al. ${ }^{[8]}$ applied X-CT to detect the internal defects of Hastelloy fabricated by selective laser melting (SLM). It was found that the evaluation ability of X-CT was limited by the thickness of the sample. UT often needs algorithm processing due to the 
influence of noise ${ }^{[9]}$. Off-line detection methods are relatively mature, but still cannot effectively detect the internal defects in thick and large parts, nor can they provide feedback and adjust the AM process in-situ. Therefore, it is necessary to develop an in-situ detection technology. Some detection techniques based on optics, such as a charge coupled device (CCD) camera, digital single lens reflex (DSLR) camera and high-speed camera, have been applied to on-line inspection in the process of $\mathrm{AM}{ }^{[10-12]}$. Although the on-line detection of optical testing technology is relatively mature, it cannot detect interlayer defects. In-situ X-ray imaging method can monitor in real time the evolution of molten pool and keyhole formation in the PBF process, which contributes to the understanding of the dynamics in the PBF process ${ }^{[13,14]}$. However, high-speed X-ray imaging technology is not applicable to industrial sites due to its high cost.

Laser ultrasonic testing (LUT) uses laser to generate and detect an ultrasonic wave, which is a non-destructive and non-contact testing technology. It is found that shear wave, longitudinal wave, and Rayleigh wave are generated when laser interacts with the material surface due to thermoelastic effect ${ }^{[15,16]}$. LUT can function under the complex environments of radiation, temperature, pressure and corrosion. Therefore, LUT has shown great potential in the on-line inspection of AM. At present, some researchers have applied LUT to the detection of AM samples. Zhan et al. ${ }^{[17]}$ applied laser ultrasound to evaluate the residual stress of AM samples under different heat treatment processes. Ma et al. ${ }^{[18]}$ investigated a laser opto-ultrasonic dual detection technology for testing elemental compositions and residual stress of wire + arc additive manufacturing sample. Everton et al. ${ }^{[19]}$ inspected a AM Ti-6Al-4V sample with subsurface defects by LUT. It was found that the location of the defects was revealed by B-scan image. Millon et al. ${ }^{[20]}$ used linear laser to generate Rayleigh wave to detect the defects of AM sample. It was found the location of surface crack-type defects $0.2 \mathrm{~mm}$ wide can be identified according to the B-scan image. Smith et al. ${ }^{[21]}$ detected and identified surface and near-surface defects with diameters of about $100 \mu \mathrm{m}$ by spatially resolved acoustic spectroscopy (SRAS). However, SRAS is only suitable for samples with very smooth surfaces. Davis et al. ${ }^{[22]}$ utilized longitudinal waves to detect an AM part with an LUT device, and hole defects with diameters more than $2 \mathrm{~mm}$ were identified according to the B-scan and C-scan images. However, their experimental method is not suitable for the on-line testing of AM parts because the surface of the AM sample used is ground smooth. In fact, the effect of surface roughness on the signal-to-noise ratio (SNR) of laser ultrasonic signals is an important factor affecting the realization of LUT on-line inspection.

In this study, an LUT system approaching the actual environment of on-line detection was established to detect near-surface defects of a 316L stainless steel sample fabricated by L-PBF using laser ultrasonic wave. The capability of LUT for on-line inspection of L-PBF process was evaluated, and a hypothetical scheme of an on-line detection integrated with the AM system was proposed.

\section{Experimental methods}

\subsection{Sample fabrication}

In this work, the sample of $316 \mathrm{~L}$ stainless steel was fabricated using L-PBF equipment (NCL-M2150T). The L-PBF process parameters of prepared sample are: laser power of $280 \mathrm{~W}$, scanning speed of $1,200 \mathrm{~mm} \cdot \mathrm{s}^{-1}$, hatch spacing of $0.1 \mathrm{~mm}$, and layer thickness of $30 \mu \mathrm{m}$. Six near-surface defects with different depths in the sample were machined by electrical discharge machining (EDM). The defects were designed to be narrow and elongated in shape to mimic typical internal cracks in an AM product. Figure 1 shows the dimensions and locations of the samples and preset defects. A surface roughness measuring instrument (TIME 3221) and stereomicroscope (SZ6100) were used to evaluate the surface quality of the AM sample.
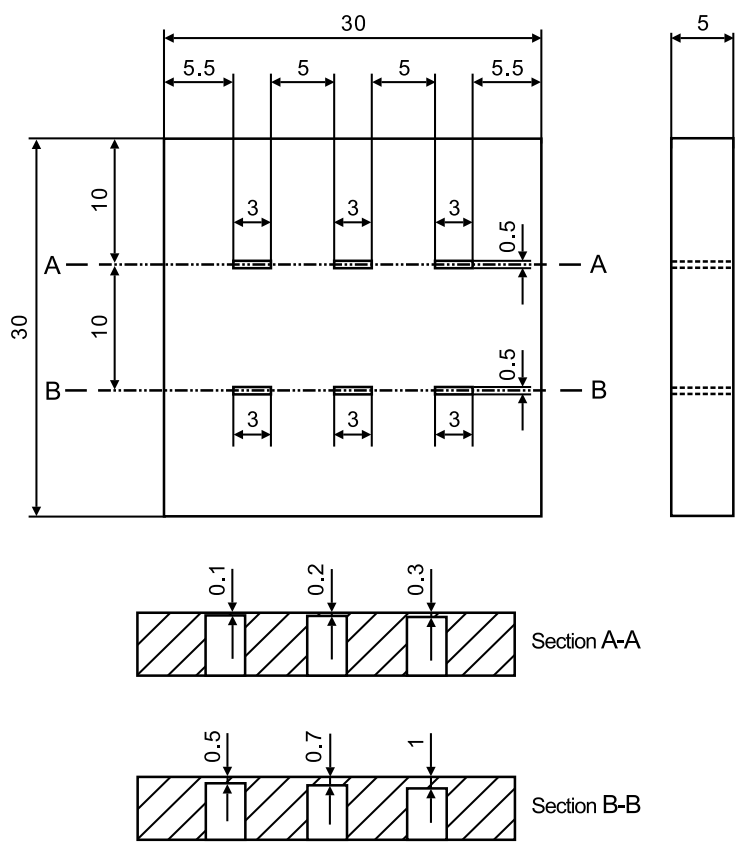

Fig. 1: Schematic of dimensions and locations of sample and preset defects

\subsection{Laser ultrasonic testing}

As shown in Fig. 2, an LUT system was established for the testing of the AM samples. The pulse laser (WEDGE 1064 HB DB) with a wavelength of 1,064 nm was used for the generation of an ultrasonic wave based on the thermoelastic effect. The size of the focal spot diameter for the generation laser is $100 \mu \mathrm{m}$. The laser ultrasonic receiver (QUARTET-1500) was used to receive ultrasonic signals, which used a continuous laser with a wavelength of $532 \mathrm{~nm}$. The detail parameters of the LUT system are shown in Table 1 . The positioning system was used to realize the synchronous scanning of the generation laser and the detection laser. A data acquisition system and computer were used to collect and process the data.

As shown in Fig. 3, the generation laser point and the detection laser point were focused on the surface of sample and kept fixed distance of $2 \mathrm{~mm}$. The scanning path was parallel to the $x$-axis. The sample was placed on the motorized 
(a)

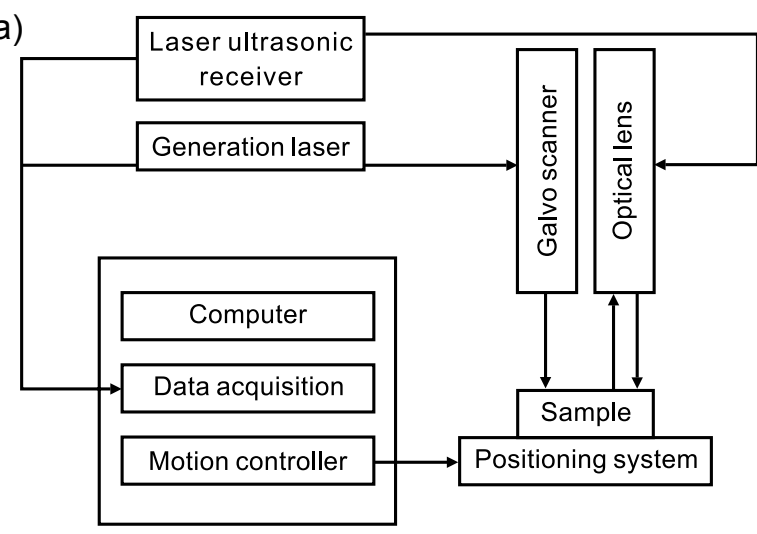

(b)

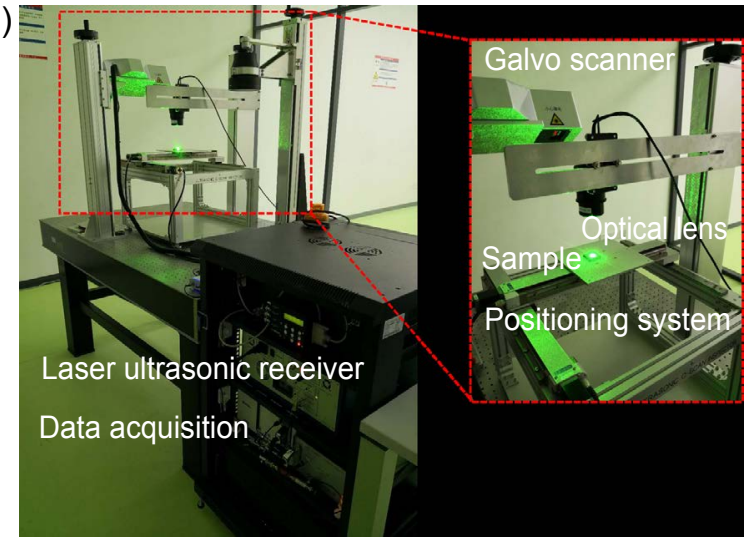

Fig. 2: System of LUT: (a) schematic diagram; (b) experimental device

Table 1: Detail parameters of LUT system

$\begin{array}{cccc}\text { Parameters of generation laser } & \text { Value } & \text { Parameters of detection laser } & \text { Value } \\ \text { Wavelength }(\mathrm{nm}) & 1,064 & \text { Wavelength }(\mathrm{nm}) & 532 \\ \text { Output energy }(\mathrm{mJ}) & \text { Up to } 2 & \text { Laser diameter }(\mu \mathrm{m}) & 50 \\ \text { Pulse width }(\mathrm{ns}) & 1.6 & \text { Laser power }(\mathrm{W}) & 1.5 \\ \text { Repetition frequency }(\mathrm{Hz}) & 500 & \text { Testing bandwidth }(\mathrm{MHz}) & 0.19-102\end{array}$

(a)

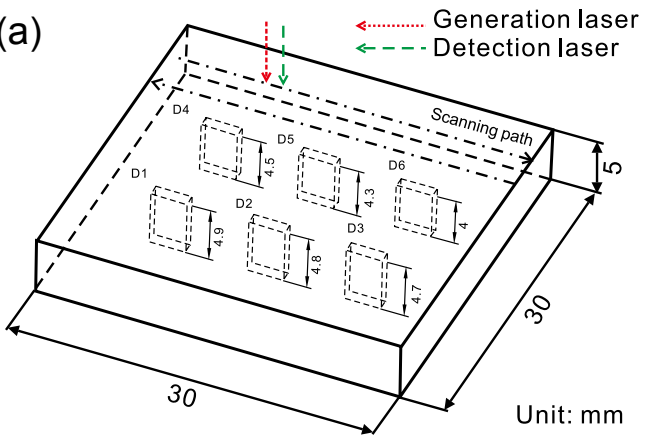

(b)

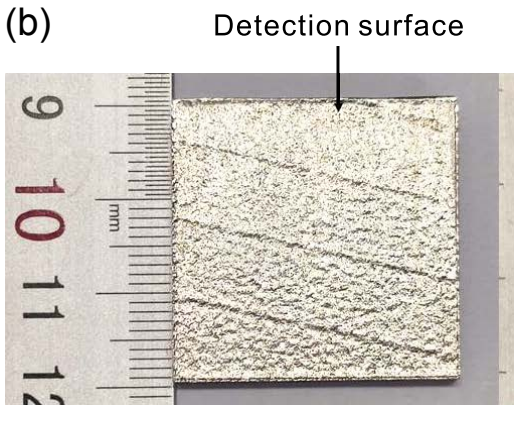

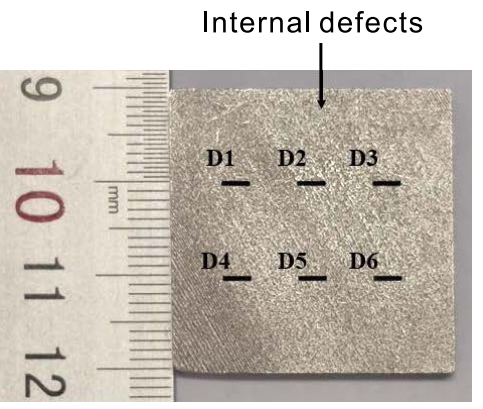

Fig. 3: Inspection scheme and L-PBF 316L sample: (a) CAD model of sample and inspection scheme; (b) surface of both sides of investigated sample

positioning system and scanning was realized by the positioning system. The distance between adjacent detection points in the $x$ direction $(\mathrm{d} x)$ and $y$ direction $(\mathrm{d} y)$ was $0.1 \mathrm{~mm}$. The scanning area was $20 \mathrm{~mm} \times 25 \mathrm{~mm}$, covering all the defects, for a total of 50,451 scan points. Since the fastest scanning speed of the galvanometer was 500 points per second, it only took $100 \mathrm{~s}$ to complete the scanning of the $20 \mathrm{~mm} \times 25 \mathrm{~mm}$ region if the movement of the excitation laser and detection laser were controlled by the galvanometer. Of course, $0.1 \mathrm{~mm}$ is a smaller scan spacing, mainly used for accurate detection of tiny defects. The actual scan spacing for normal detection should be larger than this. The large scan spacing is more efficient, but the detection resolution is low.

\subsection{X-ray computed tomography}

An X-ray computed tomography (Y. CT Precision) scan was used to fully characterize the defects of the sample in order to verify the experimental results of the LUT system. The defects in the sample would be shown by the gray-scale diagram based on the difference in X-ray absorption of the sample. The specific parameters of the testing are shown in Table 2.

Table 2: Specific parameters of X-CT

\begin{tabular}{|cc|}
\hline Parameter & Value \\
\hline Voltage $(\mathrm{kV})$ & 195 \\
Current $(\mathrm{mA})$ & 0.3 \\
Detector type & Y.XRD0820 \\
Number of detector elements & 1,024 \\
Resolution $(\mu \mathrm{m})$ & 40 \\
\hline
\end{tabular}




\section{Results and discussion}

\subsection{Influence of surface roughness}

Due to the influence of surface roughness on the signal-tonoise ratio (SNR) of LUT, the signal of ultrasonic wave was obtained on the smooth and rough AM samples respectively. The results of the roughness testing of the sample surface are shown in Fig. 4. Five areas [Fig. 4(a)] are selected on the surface of the sample for roughness testing, and the average roughness is $7.45 \pm 0.9 \mu \mathrm{m}$. Figure 4 (b) shows the surface morphology of the sample. It is found that the surface of the L-PBF sample exhibited lots of directional stripes and balling phenomenon, which is related to the scanning path in the L-PBF process.
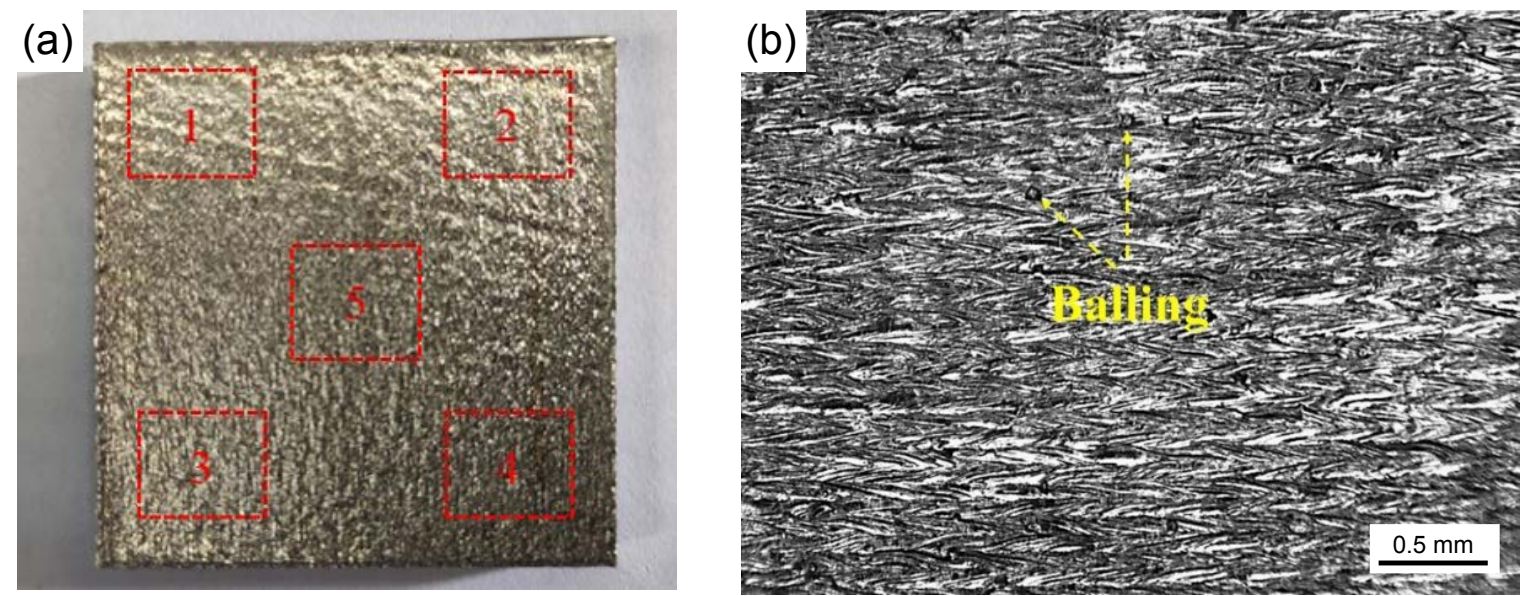

Fig. 4: Sample roughness: (a) testing area; (b) surface morphology of sample

Another L-PBF sample was ground to a smooth surface with $\mathrm{Ra}=0.4 \mu \mathrm{m}$. The laser ultrasonic A-scan signals were collected on the surfaces of the samples with $\mathrm{Ra}=7.45 \mu \mathrm{m}$ and $\mathrm{Ra}=0.4 \mu \mathrm{m}$ with same parameters of LUT, respectively. The results are shown in Fig. 5. According to the formula $\mathrm{SNR}=201 \mathrm{~g}(A / \sigma)^{[23]}$, where $A$ is the signal's strength and $\sigma$ is the standard deviation of the noise. The average SNR of multiple sets of laser ultrasonic signals collected on smooth and rough surfaces was calculated respectively. The average SNR of the Rayleigh wave signal for the smooth surface is $25.80 \mathrm{~dB}$, and that for the rough surface is $18.64 \mathrm{~dB}$, much lower than the smooth sample. The main reason is that the rough surface creates diffuse reflection to the detection laser, which reduces the sensitivity of the laser ultrasonic receiver.
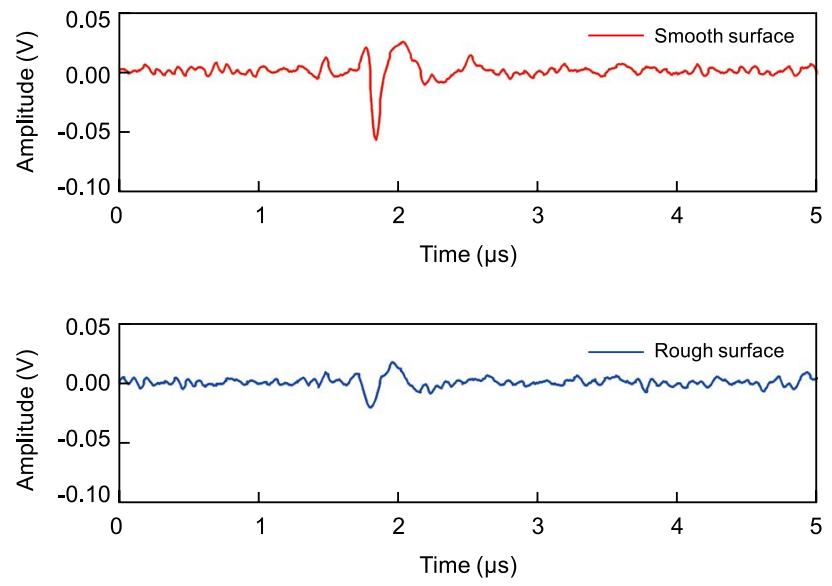

Fig. 5: A-scan signals of Rayleigh wave under conditions of smooth surface and rough surface

\subsection{Laser ultrasonic testing}

In LUT, an A-scan image shows the voltage amplitude of the ultrasonic signals as a function of time at a specific detection point. A B-scan image can be generated by processing all the A-scan signals on a line parallel to the $x$-axis or $y$-axis. In a B-scan image, the $x$-axis represents the spatial location, the $y$-axis represents time, and the signal amplitude is denoted by color. A C-scan image is obtained by mapping all the A-scan signals in a scan area. The positions $x$ and $y$, as well as the time $t$ form a three-dimensional array $(x, y, t)$ and the amplitude of signals is represented by color.

LUT was carried out on the AM 316L sample with artificial defects of different depths. Figure 6 shows A-scan signal images generated by the excitation laser at locations far away, near and above the near-surface defect of depth of $0.1 \mathrm{~mm}$, respectively. As shown in Fig. 6(a), when the excitation laser is far away from the near-surface defect, the direct Rayleigh wave is detected. In Fig. 6(b), the phase of Rayleigh wave evolves when the excitation laser approaches the near-surface defect, marked by the red arrow in the inset. This phenomenon is mainly attributed to the superimposition of the reflected wave and direct Rayleigh wave ${ }^{[24]}$. In Fig. 6(c), when the excitation laser is above the near-surface defect, the Lamb wave is detected. This phenomenon is mainly attributed to the upper part of the near-surface defect being equivalent to a plate thickness of $0.1 \mathrm{~mm}$. The spectrum of Rayleigh wave signal is shown in Fig. 7. It can be found that the bandwidth of the surface wave is in the range of 2-4 MHz. The sound velocity of the surface wave measured by experiment is $3,021 \mathrm{~m} \cdot \mathrm{s}^{-1}$, so the wavelength of the Rayleigh wave is $0.6-1.3 \mathrm{~mm}$. 
(a)

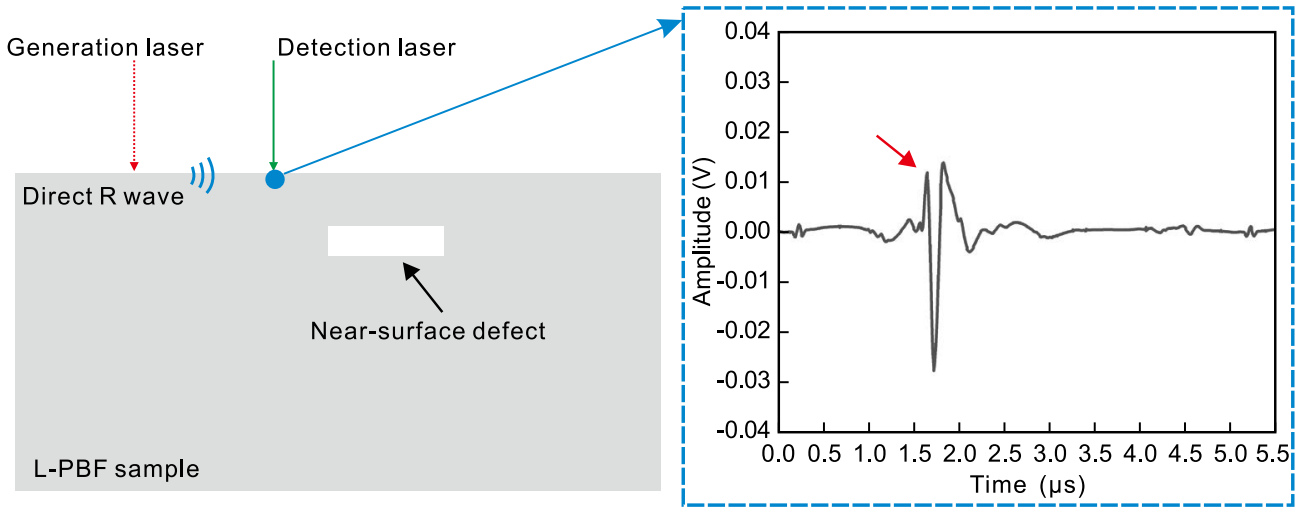

(b)

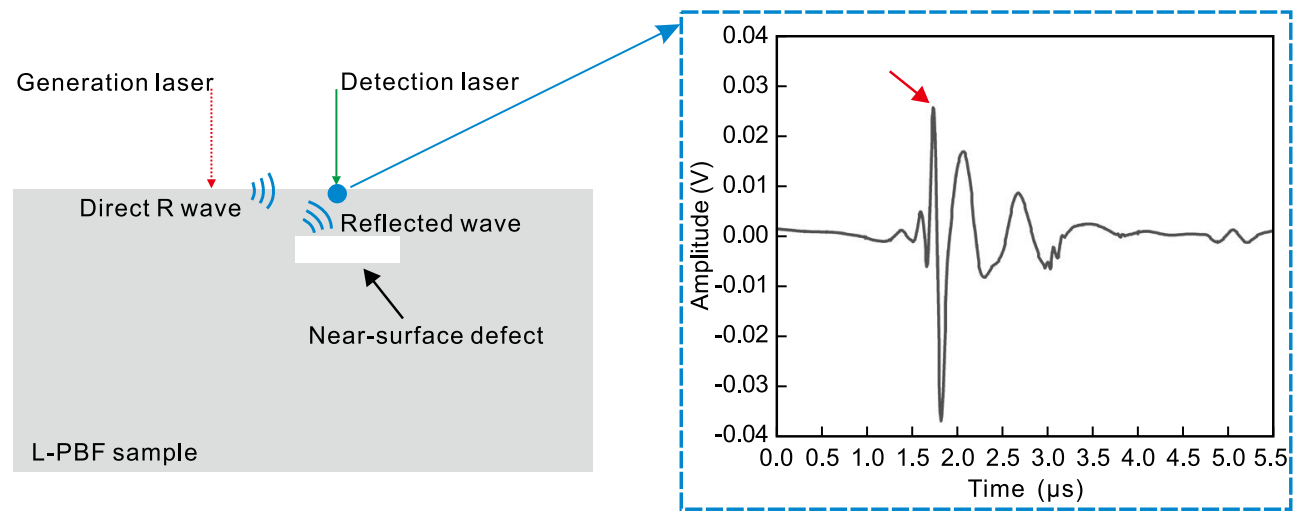

(c)

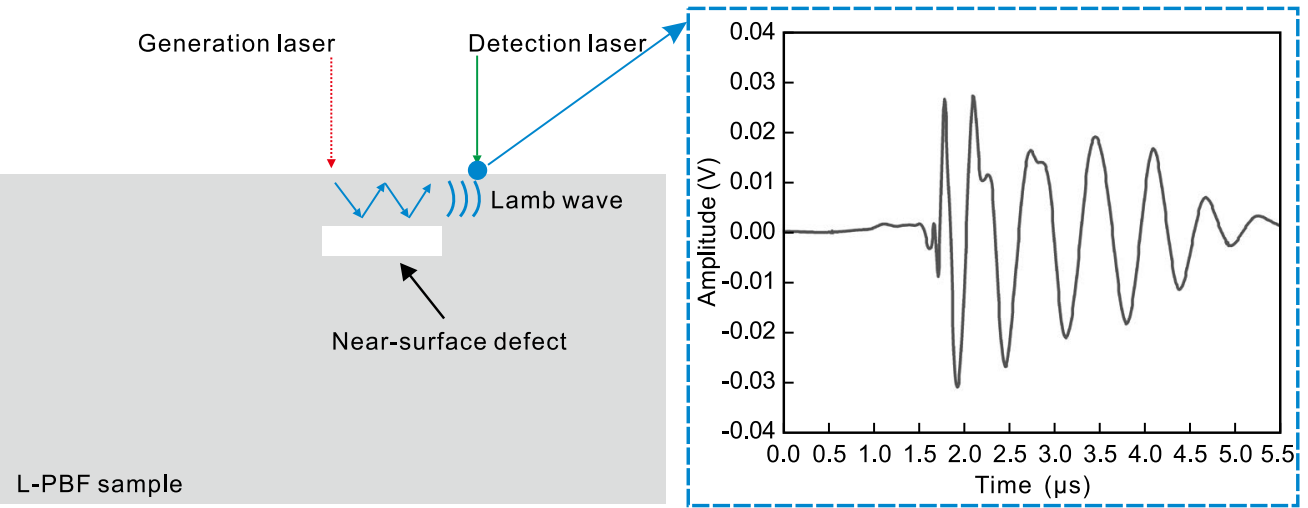

Fig. 6: A-scan signal images generated by excitation laser at locations far away (a), near (b) and above (c) near-surface defect of depth of $0.1 \mathrm{~mm}$

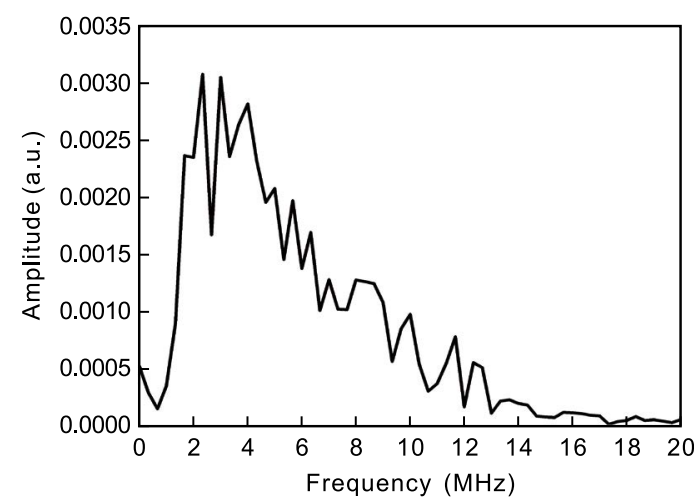

Fig. 7: Spectrum of A-scan signal at location without near-surface defect

Figure 8 shows the A-scan signals obtained when the excitation laser is above the near-surface defect at different depths. It is found that the vibration signal gradually changes from Lamb wave to Rayleigh wave as the depth of the defect increases. This is mainly because the Lamb wave is generated by excitation laser when the depth of the defect is less than the wavelength $(0.6-1.3 \mathrm{~mm})$ of the Rayleigh wave, and the Rayleigh wave is generated by excitation laser when the depth of the defect is greater than the wavelength of the Rayleigh wave.

Figures 9(a) and (b) depict the B-scan results along the $x$-direction at locations of $y=10 \mathrm{~mm}$ and $20 \mathrm{~mm}$, also showing the existence of near-surface defects. From the B-scan results, the wavefront of Rayleigh wave appears at approximately $t=1.8 \mu \mathrm{s}$. When a near-surface defect is present, a striated area appears in B-scan image due to the generation of Lamb wave. In addition, the length of the striated area is about $3 \mathrm{~mm}$, which is consistent with the length of the preset defects in $x$-direction. When the depth of the defect reaches $0.7 \mathrm{~mm}$, the striated area is almost invisible in the image, which means that the ultrasonic waves generated by laser changed from Lamb wave to Rayleigh wave. 

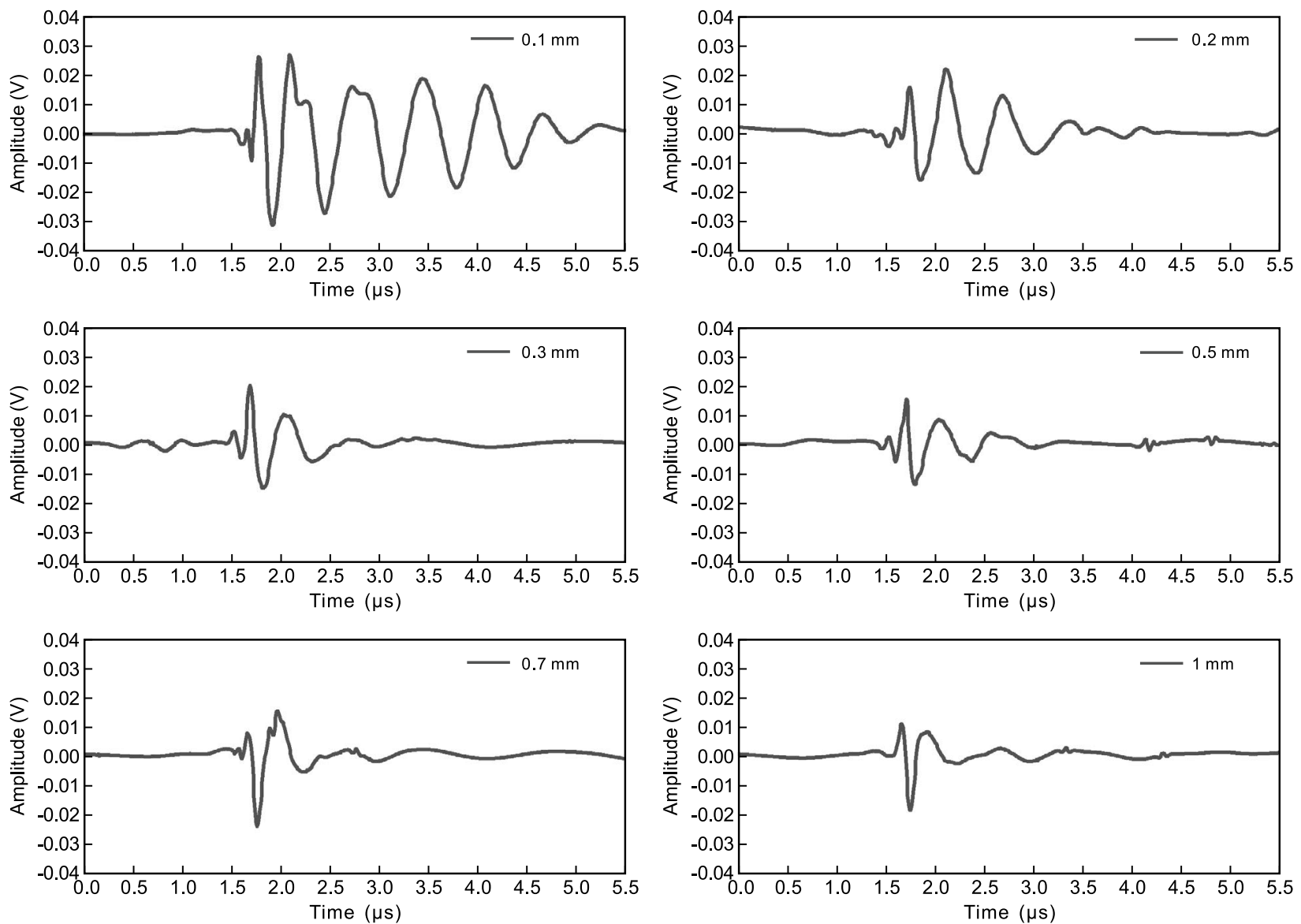

Fig. 8: A-scan signals at near-surface defects of different depths
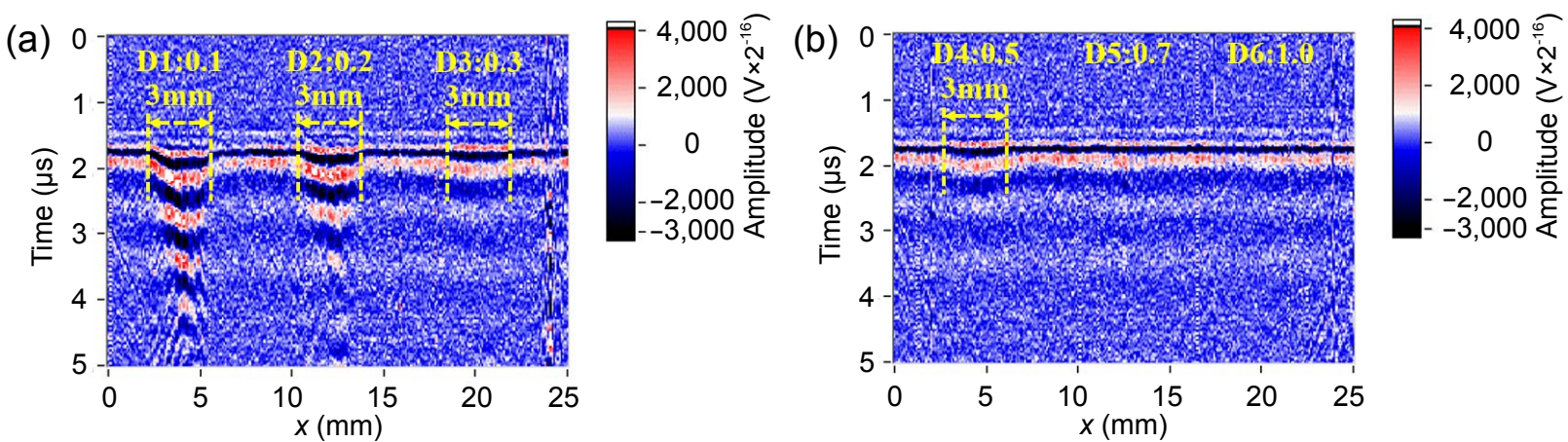

Fig. 9: B-scan image indicating presence of near-surface defects: (a) $y=10 \mathrm{~mm}$; (b) $y=20 \mathrm{~mm}$

Figure 10 is the C-scan result of LUT. Figure $10(\mathrm{~b})$ is the result after median filtering and threshold processing of (a). It is found there is lots of background noise in C-scan image, which is due to the low SNR caused by the surface roughness of the AM sample. The location of near-surface defects with depths of $0.1 \mathrm{~mm}, 0.2 \mathrm{~mm}, 0.3 \mathrm{~mm}$ and $0.5 \mathrm{~mm}$ can be identified clearly. However, near-surface defects with depths of $0.7 \mathrm{~mm}$ and $1 \mathrm{~mm}$ are difficult to distinguish from the background. In addition, the approximate shape and size of the detected defects can also be identified from the C-scan image, which correspond to the actual defect shape and size.

\subsection{X-ray computed tomography}

The results of the X-CT test are shown in Fig. 11. The 6 near- surface defects with different depths are identified clearly. The $\mathrm{X}-\mathrm{CT}$ results also show a dense AM sample with few pores. Table 3 shows the results of defect size tested by LUT and $\mathrm{X}$-CT respectively, showing a maximum error of $10 \%$ in defect length and $17 \%$ in width using the LUT method. Comparing $\mathrm{X}-\mathrm{CT}$ and LUT, although LUT can only detect the near-surface defects within the depth of $0.5 \mathrm{~mm}$ from the AM sample surface, the results of detection, including position, shape and size of the defects, are close to the actual ones. Despite the higher error of LUT compared with X-CT, considering its convenience and potential for on-line testing, the LUT approach has sufficient accuracy to realize semi-quantitative detection of the sub-millimeter near-surface defects on an AM surface with a roughness of $\mathrm{Ra}=7.45 \mu \mathrm{m}$. 

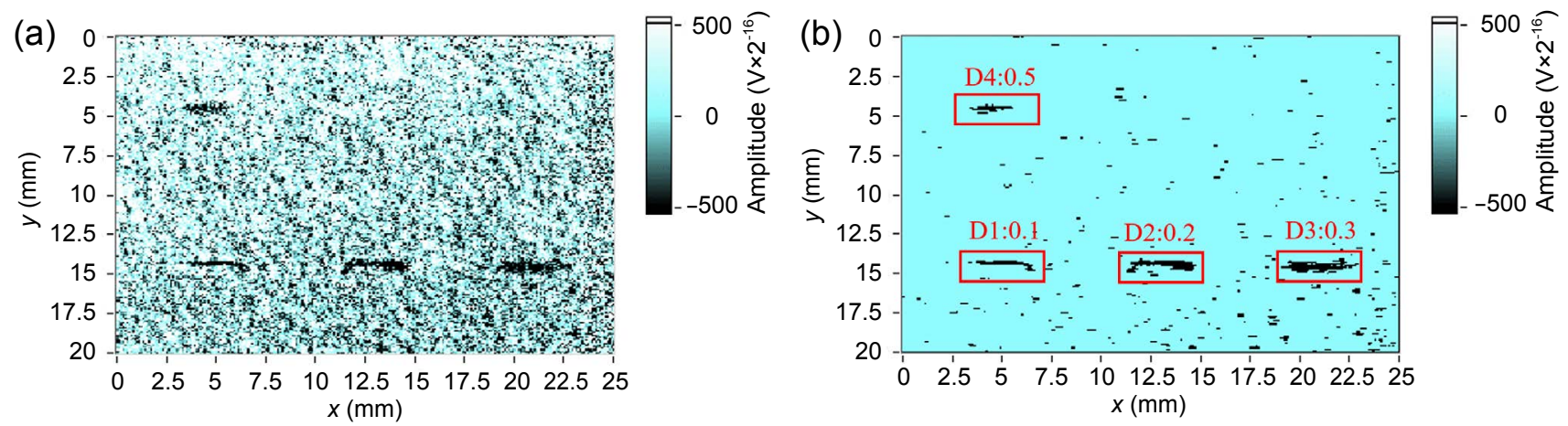

Fig. 10: C-scan images demonstrating near-surface defects

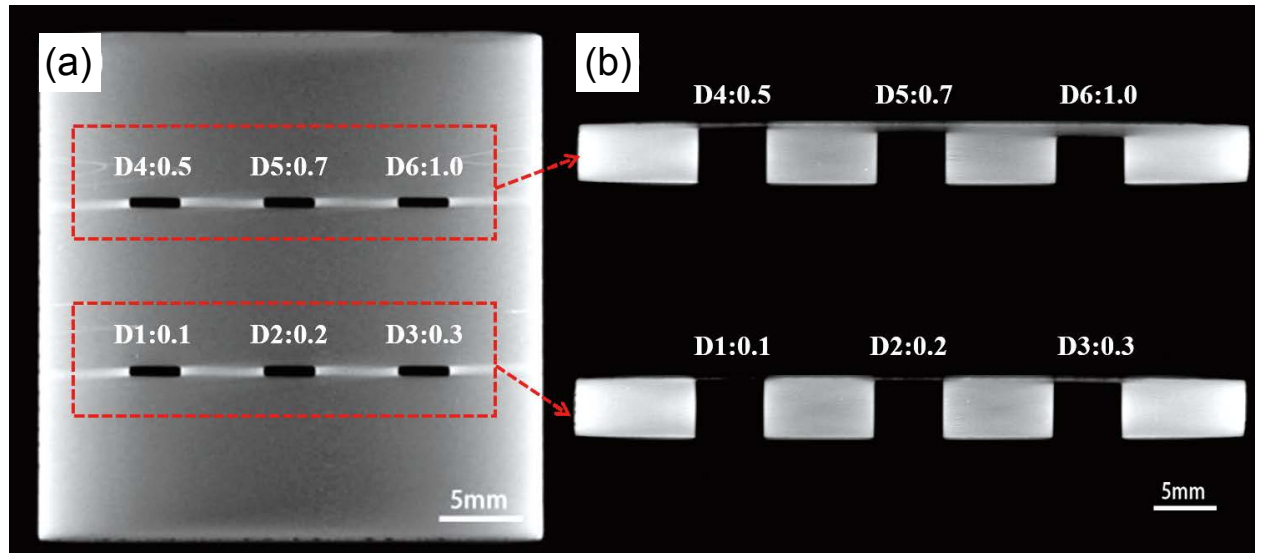

Fig. 11: Experimental results of X-CT: (a) sectional top view of defects; (b) sectional front view of defects

Table 3: Comparison of results of X-CT and LUT

\begin{tabular}{|c|c|c|c|c|c|}
\hline Defects & $\begin{array}{l}\text { Depth } \\
(\mathrm{mm})\end{array}$ & $\begin{array}{l}\text { X-CT result of length } \\
(\mathrm{mm})\end{array}$ & $\begin{array}{l}\text { X-CT result of width } \\
(\mathrm{mm})\end{array}$ & $\begin{array}{l}\text { LUT result of length } \\
(\mathrm{mm})\end{array}$ & $\begin{array}{l}\text { LUT result of width } \\
(\mathrm{mm})\end{array}$ \\
\hline D1 & 0.1 & 3.00 & 0.58 & 2.8 & 0.4 \\
\hline D2 & 0.2 & 3.00 & 0.58 & 2.9 & 0.5 \\
\hline D3 & 0.3 & 3.00 & 0.58 & 3.3 & 0.6 \\
\hline D4 & 0.5 & 3.00 & 0.63 & 2.8 & 0.6 \\
\hline D5 & 0.7 & 3.00 & 0.63 & Failed to detect & Failed to detect \\
\hline D6 & 1.0 & 3.00 & 0.63 & Failed to detect & Failed to detect \\
\hline
\end{tabular}

\subsection{On-line detection implementation strategy}

The principles of the hypothetical scheme are shown in Fig. 12, showing how the LUT system can work with an additive manufacturing system to implement on-line detection. From Fig. 12, the realization of on-line detection is accomplished by alternating AM processes and LUT processes. In each AM process, powders are melted by the heat source and accumulate layer by layer for several layers and defects may form in the meantime. The layer accumulation thickness in this round of AM process should be within a range that the next LUT can cover. For instance, the thickness of 10 layers is typically $200-500 \mu \mathrm{m}$ depending on the particle size of the powder used. After a round of AM process, the detection scan is conducted by the LUT system before the next round of AM process starts. During the LUT process, using an excitation laser and a detection laser projecting onto the as-printing surface, the inspection data of A-scan, B-scan, and C-scan with information of defects are acquired. Once the whole AM process is complete, the defects distribution in the finished part can be reconstructed by parsing the LUT data. To reduce the modification of additive manufacturing system during equipment integration, the schematic diagram of the equipment integration scheme is shown in Fig. 12(b), where an on-line LUT system can be integrated with a $3 \mathrm{D}$ printing system by sharing the galvanometer with the printing laser, adding a 
(a)
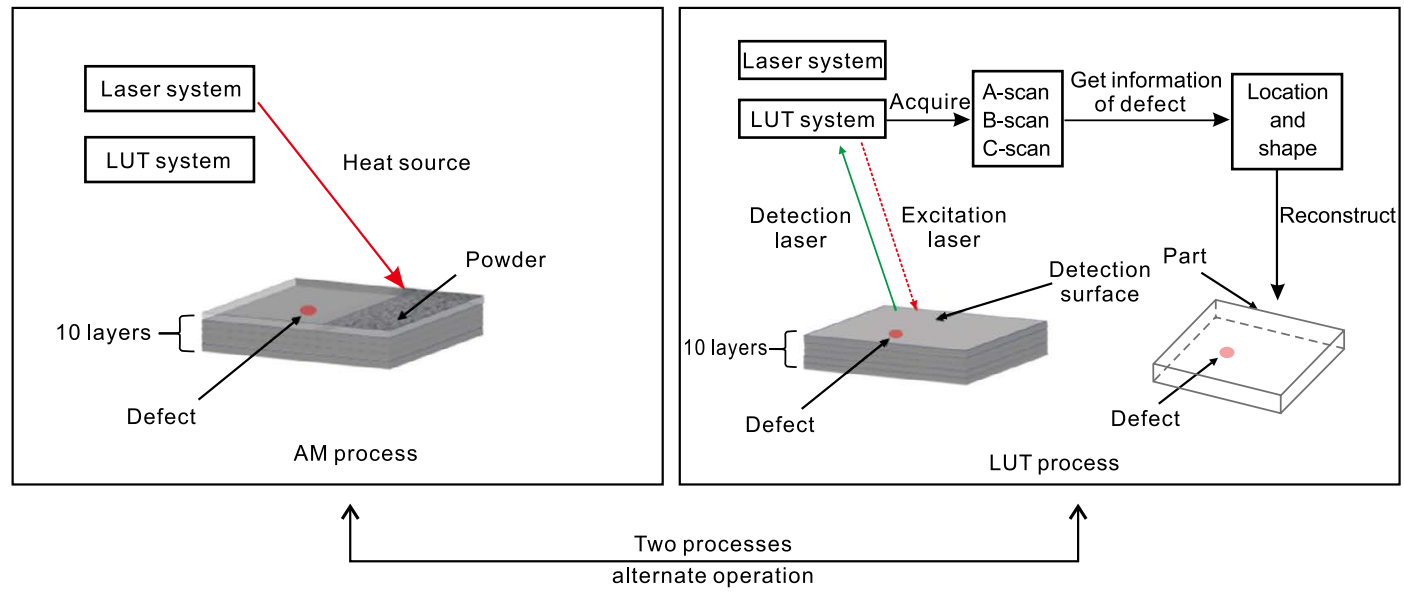

(b)

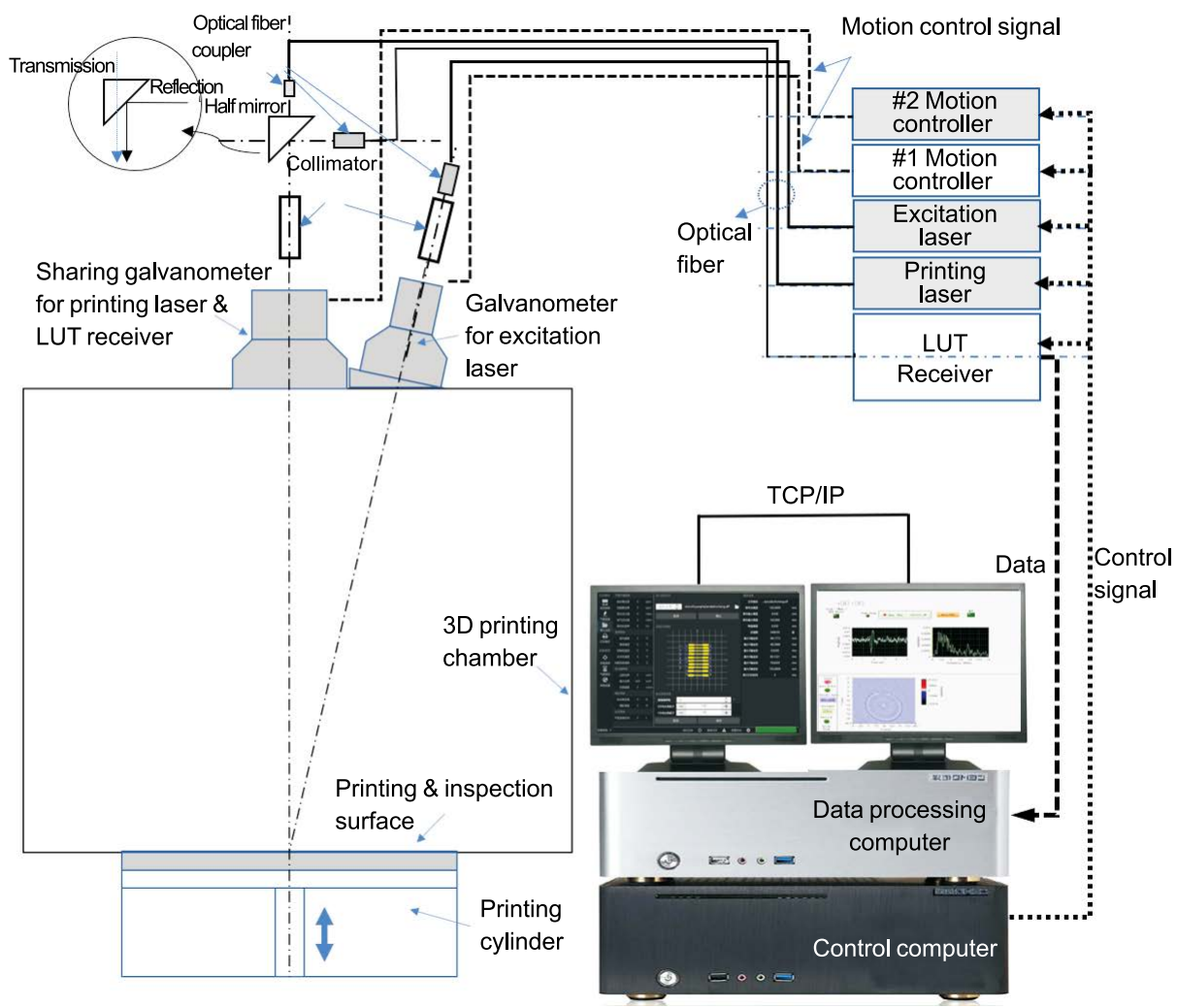

Fig. 12: Schematic of LUT method for on-line inspection in L-PBF: (a) schematic of on-line LUT method; (b) schematic of equipment integration scheme

galvanometer for excitation laser, and related optical parts, controller and data acquisition and processing units. The data communication between two industrial personal computers (IPC) is realized through TCP/IP. Of course, in the process of on-line detection, the influence of temperature on ultrasonic signal, the coordination of the printing paths and the testing paths, the noise reduction processing, and the fast imaging of the testing data all need to be considered, and further study is needed.

\section{Conclusions}

In this research, a system of LUT was established approaching the actual environment of on-line detection, and the applicability of the LUT system for defects detection of L-PBF parts was evaluated, and was validated by X-CT. The effectiveness of the LUT technology to achieve on-line testing of L-PBF was also evaluated. The main conclusions are listed as follows:

(1) The surface roughness of the AM sample causes the severe reduction of the SNR of the LUT A-scan signal. Compared with the smooth surface with Ra of $0.4 \mu \mathrm{m}$, the SNR of Rayleigh wave decreases by $7.16 \mathrm{~dB}$ from $25.80 \mathrm{~dB}$ on the AM rough surface with Ra of $7.45 \mu \mathrm{m}$.

(2) When the excitation laser encounters a near-surface defect with a low depth during scanning, it will generate a Lamb wave, which is conducive to quantitative analysis of the position and size of the defect. 
(3) The wavelength of the surface wave determines the depth range of the defect detection. The surface wave with the wavelength of $0.6-1.3 \mathrm{~mm}$ can realize the detection of subsurface defects within the depth of $0.5 \mathrm{~mm}$.

(4) The size and position information of near-surface defects within the depth of $0.5 \mathrm{~mm}$ can be accurately identified by using the B-scan and C-scan results of the LUT system.

The LUT method exhibits great potential for on-line inspection of AM processes. However, to accomplish a balance between the acquired information and the testing efficiency of an individual LUT test, the on-line LUT strategy should be a deliberate and optimized combination of A-, Band C-scans.

\section{Acknowledgement}

This work was financially supported by the National Key R\&D Program of China (Grant No. 2018YFB1106100).

\section{References}

[1] Zhang $Y$, Wu $L M$, Guo $X Y$, et al. Additive manufacturing of metallic materials: A review. Journal of Materials Engineering and Performance, 2017, 27(1): 1-13.

[2] Debroy T, Wei H L, Zuback J S, et al. Additive manufacturing of metallic components - Process, structure and properties. Progress in Materials Science, 2018, 92: 112-224.

[3] Gong H J, Rafi K, Gu H F, et al. Analysis of defect generation in Ti-6Al-4V parts made using powder bed fusion additive manufacturing processes. Additive Manufacturing, 2014, 1-4: 87-98.

[4] Zhang B, Li Y T, Bai Q. Defect formation mechanisms in selective laser melting: A review. Chinese Journal of Mechanical Engineering, 2017, 30(3): 515-527.

[5] Song B, Dong S J, Zhang B C, et al. Effects of processing parameters on microstructure and mechanical property of selective laser melted Ti6Al4V. Materials \& Design, 2012, 35: $120-125$.

[6] Ziółkowski G, Chlebus E, Szymczyk P, et al. Application of X-ray CT method for discontinuity and porosity detection in 316L stainless steel parts produced with SLM technology. Archives of Civil and Mechanical Engineering, 2014, 14(4): 608-614.

[7] Sol T, Hayun S, Noiman D, et al. Nondestructive ultrasonic evaluation of additively manufactured AISi $10 \mathrm{Mg}$ samples. Additive Manufacturing, 2018, 22: 700-707.

[8] Rometsch Paul A, Pelliccia D, Tomus D, et al. Evaluation of polychromatic $\mathrm{X}$-ray radiography defect detection limits in a sample fabricated from Hastelloy $X$ by selective laser melting. NDT \& E International, 2014, 62: 184-192.
[9] Song Y F, Zi X H, Fu Y D, et al. Nondestructive testing of additively manufactured material based on ultrasonic scattering measurement. Measurement, 2018, 118: 105-112.

[10] Lott P, Schleifenbaum H, Meiners W, et al. Design of an optical system for the in situ process monitoring of selective laser melting (SLM). Physics Procedia, 2011, 12: 683-690.

[11] Repossini G, Laguzza V, Grasso M, et al. On the use of spatter signature for in-situ monitoring of laser powder bed fusion. Additive Manufacturing, 2017, 16: 35-48.

[12] Lu Q Y, Nguyen N V, Hum A J W, et al. Optical in-situ monitoring and correlation of density and mechanical properties of stainless steel parts produced by selective laser melting process based on varied energy density. Journal of Materials Processing Technology, 2019, 271: 520-531.

[13] Zhao C, Fezzaa K, Cunningham R W, et al. Real-time monitoring of laser powder bed fusion process using high-speed X-ray imaging and diffraction. Scientific Reports, 2017, 7(1): 3602.

[14] Leung C L A, Marussi S, Atwood R C, et al. In situ X-ray imaging of defect and molten pool dynamics in laser additive manufacturing. Nature Communication, 2018, 9(1): 1355.

[15] Zeng W, Yao Y Y, Qi S K, et al. Finite element simulation of laser-generated surface acoustic wave for identification of subsurface defects. Optik, 2020, 207: 163812.

[16] Choi S, Jhang K Y. Internal defect detection using lasergenerated longitudinal waves in ablation regime. Journal of Mechanical Science and Technology, 2018, 32(9): 4191-4200.

[17] Zhan Y, Xu H X, Du W Q, et al. Research on the influence of heat treatment on residual stress of TC4 alloy produced by laser additive manufacturing based on laser ultrasonic technique. Ultrasonics, 2021, 115: 106466.

[18] Ma Y Y, Hu Z L, Tang Y, et al. Laser opto-ultrasonic dual detection for simultaneous compositional, structural, and stress analyses for wire + arc additive manufacturing. Additive Manufacturing, 2020, 31: 100956.

[19] Everton S, Dickens P, Tuck C, et al. Using laser ultrasound to detect subsurface defects in metal laser powder bed fusion components. JOM, 2018, 70(3): 378-383.

[20] Millon C, Vanhoye A, Obaton A F, et al. Development of laser ultrasonics inspection for online monitoring of additive manufacturing. Welding in the World, 2018, 62(3): 653-661.

[21] Smith R J, Hirsch M, Patel R, et al. Spatially resolved acoustic spectroscopy for selective laser melting. Journal of Materials Processing Technology, 2016, 236: 93-102.

[22] Davis G, Nagarajah R, Palanisamy S, et al. Laser ultrasonic inspection of additive manufactured components. The International Journal of Advanced Manufacturing Technology, 2019, 102(5-8): 2571-2579.

[23] Truong T C, Lee J R. SNR enhancement for composite application using multiple Doppler vibrometers based laser ultrasonic propagation imager. Optics \& Lasers in Engineering, 2016, 84: 82-88.

[24] Chen D, Lü G L, Guo S F, et al. Subsurface defect detection using phase evolution of line laser-generated Rayleigh waves. Optics \& Laser Technology, 2020, 131: 106410. 